\title{
Matching theory based user-grouping for indoor non-orthogonal multiple access visible light communication heterogeneous networks
}

\author{
Zhengxi Chen (陈政希), Zhitong Huang (黄治同)*, and Yuefeng Ji (纪越峰) \\ State Key Laboratory of Information Photonics and Optical Communications, School of Information and \\ Communication Engineering, Beijing University of Posts and Telecommunications, Beijing 100876, China \\ *Corresponding author: hzt@bupt.edu.cn
}

Received December 6, 2019; accepted March 1, 2020; posted online May 12, 2020

\begin{abstract}
This Letter proposes a model of indoor visible light communication (VLC) heterogeneous networks entirely based on LEDs with different specifications and applies non-orthogonal multiple access (NOMA) to it because of the narrow modulation bandwidth of LEDs. Moreover, a user-grouping scheme that is based on matching theory is proposed to improve the network achievable sum rate. Simulation results indicate that when each NOMA cluster contains 6 users, the proposed scheme has a $49.54 \%$ sum-rate enhancement compared with the traditional user-grouping scheme. As the number of users in each NOMA cluster increases, the proposed scheme performs better at the cost of computational complexity.

Keywords: visible light communication; non-orthogonal multiple access; matching theory; user grouping.

doi: 10.3788/COL202018.060602.
\end{abstract}

With the development of $5 \mathrm{G}$ technology, various smart devices such as 'Internet of Things', virtual reality, and smart cities have emerged. The convergence of optical communication and wireless communication is an important development trend for the flexible carrying of large capacity services ${ }^{[1,2]}$. Visible light communication (VLC), as a typical optical communication system capable of supporting next-generation high-speed wireless networks, has attracted widespread attention in academia and industry - [3.4] Especially in recent years, the research on underwater VLC systems ${ }^{[5,6]}$ has opened up a new area, and visible light positioning also has made further progress ${ }^{[]}$. VLC uses light-emitting diodes (LEDs) as transmitters and photodiodes (PDs) as receivers to transmit data at the frequency that human eyes are not able to perceive. Because light waves are also a kind of electromagnetic waves, they have many similarities with radio waves. However, the terahertz-level spectrum occupied by visible light has not been applied to wireless communication, so it can effectively alleviate the spectrum congestion problem occurring at the current radio frequencies ${ }^{[8]}$. Furthermore, the VLC network that is based on LEDs is an additional network layer in the existing heterogeneous wireless networks that does not interfere with radio frequencies, which makes the VLC have a bright prospect for development in some electromagnetic interference sensitive environments ${ }^{[9]}$.

In order to achieve a faster rate and a more comprehensive coverage, the ultra-density of small cells is an inevitable trend in future VLC networks. Compared with the traditional communication networks, the characteristic that visible light cannot penetrate walls allows it to achieve spatial multiplexing in adjacent communication units, which can further reduce the cell size compared to the femtocell network corresponding to radio frequencies $\frac{[10]}{-}$. In an indoor environment, there are not only roof lights but also other light sources such as table lamps. In some large public places such as airports and train stations, the light source is more complicated. The complex indoor light environment also indicates that the research on VLC heterogeneous networks (HetNets) is of great importance.

Traditional orthogonal multiple access techniques, such as time-division multiple access and frequency-division multiple access, orthogonalize resources so that interference is effectively suppressed. However, since each resource block can only accommodate one user, this limits the number of users that can be accessed, which is a bottleneck for the goal of $5 \mathrm{G}$ to access a large number of users $\stackrel{[11,12]}{1}$. Non-orthogonal multiple access (NOMA), a technique to serve multiple users via one resource block, greatly improves the spectrum efficiency. Users are multiplexed in the power domain on the transmitter side by superposition coding and the signal separation is accomplished on the receiver side by successive interference cancellation (SIC) ${ }^{[11]}$. The high SNR of VLC and the relatively small number of people in the indoor environment make it suitable for the application of NOMA $\stackrel{[13]}{-}$. A study in Ref. [14] applies NOMA to indoor VLC channels and demonstrates its superior performance over orthogonal frequency-division multiple access. However, it will lead to a grouping problem owing to multiple users sharing one resource block when applying NOMA, so this Letter introduces matching theory, which has been known as an efficient technique to solve the combinatorial problem that matches players in two distinct sets. It is worth noting that matching theory can provide an appropriate model for wireless resource allocation $\frac{[15]}{-}$ and so far there is no study devoted to the implementation of matching theory on the considered visible light heterogeneous network. 
At present, most research on indoor VLC networks is mainly based on a single light source, either a homogeneous VLC network formed by multiple identical light sources $\frac{[1,17]}{1}$ or a hybrid VLC-RF system $-[18,19]$. However, there are actually many different types of light sources, such as chandeliers and desk lamps. Therefore, we propose an indoor NOMA-VLC heterogeneous network that aims to improve system performance by optimizing the matching of users within each NOMA cluster. The contribution of this Letter is that we propose a heterogeneous network based entirely on LEDs for the first time, classify users according to the accuracy of VLC in indoor positioning, and propose a NOMA-VLC user-grouping algorithm (NVUG) that applies matching theory to group users to improve the achievable sum rate of this network model.

Consider a downlink two-tier VLC heterogeneous network model in Fig. $\underline{1}$, involving one main LED (MLED) and a set of overlaid auxiliary LEDs (ALEDs), which are located within the coverage of MLED as shown in Fig. 1. The MLED is placed at height $L_{M}$ and serves $M$ main LED users (MUs). The MUs are divided into two sets according to their distance from the MLED: main LED center users (MCUs) and main LED edge users (MEUs). We denote the set of MCUs by $S_{\mathrm{MCU}}$, the $i$ th MCU by $\mathrm{MCU}_{i}, i \in\left\{1, \ldots, M_{C}\right\}$ and the set of MEUs by $S_{\mathrm{MEU}}$, the $j$ th MEU by $\mathrm{MEU}_{j}, j \in\left\{1, \ldots, M_{E}\right\}$. ALEDs deploy at height $L_{A}$, which serves $A_{L}$ auxiliary LED users (AUs), set of AUs by $S_{\mathrm{AU}}$, the lth $\mathrm{AU}$ denoted as $\mathrm{AU}_{l}$, $l \in\left\{1, \ldots, A_{L}\right\}$. Each MCU occupies one subcarrier, the MEUs need to match with one MCU to share the subcarrier, and AUs can select to be served by ALEDs or match one MCU as illustrated. Let $q_{\max }$ and $p_{\max }$, separately denote the maximum number of each MCU and each ALED they can match with.

In general, line-of-sight (LOS) and non-line-of-sight (NLOS) are both considered when studying a complete VLC channel. However, the diffuse component is at least $7 \mathrm{~dB}$ lower than the weakest LOS component received ${ }^{[20]}$, so in this Letter only the LOS part is taken into consideration. According to the Lambertian emission model, the channel gain of the optical link between the LED and the $k$ th user is given by

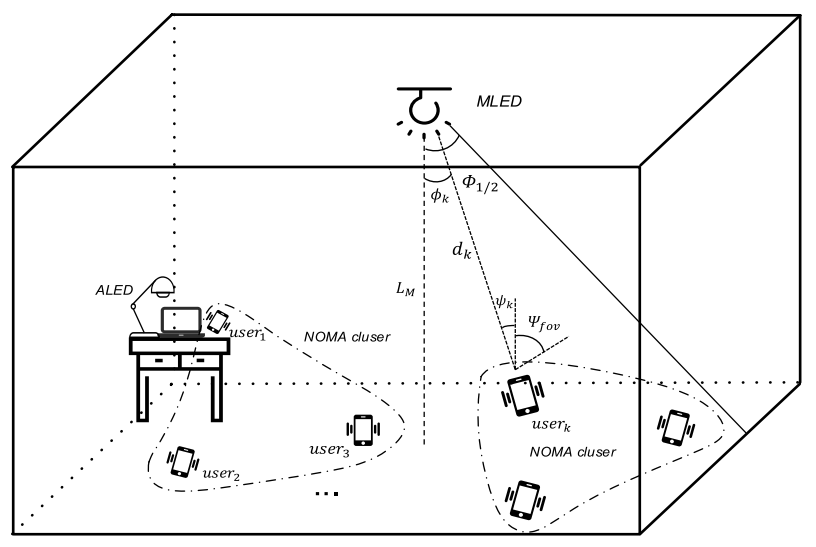

Fig. 1. Illustration of indoor NOMA-VLC HetNets.

$$
h_{k}=\frac{A R_{P}(m+1)}{2 \pi d_{k}^{2}} \cos ^{m}\left(\phi_{k}\right) T\left(\psi_{k}\right) g\left(\psi_{k}\right) \cos \left(\psi_{k}\right),
$$

where $A$ denotes the detection area of the photodiode (PD); $R_{P}$ denotes the responsivity of the PD; $d_{k}$ accounts for the distance between the LED and the $k$ th user, $T\left(\psi_{k}\right)$ represents the gain of the optical filter, and $m$ is the order of Lambertian emission relying on the transmitter semiangle $\Phi_{1 / 2}$ by $m=-\ln 2 / \ln \left(\cos \Phi_{1 / 2}\right)$. The gain of optical concentrator $g\left(\psi_{k}\right)$ is given by $n^{2} / \sin ^{2}\left(\Psi_{c}\right)$ if $0 \leq \psi_{k} \leq \Psi_{c}$ and 0 otherwise, where $\Psi_{c}$ is the concentrator field-of-view (FOV) semi-angle and $n$ is the reflective index of the optical concentrator used at the receiver front end. For visible light, the typical values for $\mathrm{n}$ are between 1 and 2 .

Assuming that the subcarrier ALEDs occupy is distinct from the subcarriers occupied by MCUs, each subcarrier uses superposition coding to transmit multiplexed signals. Let $\Phi(m)$ denote the set of users who choose subcarrier $m \in S=\left\{1, \ldots, M_{C}, M_{C}+1\right\}$. Therefore, after removing the direct current term the received signal at user $k \in \Phi(m)=\{$ user $1, \ldots$, user $K\}$ on subcarrier $m$ can be expressed as

$$
y_{k}^{m}=h_{k}^{m} s_{m}+z_{k}^{m},
$$

where $s_{m}$ indicates the superposed signal of users on subcarrier $m$, i.e., $s_{m}=\sum_{k \in \Phi(m)} \sqrt{\alpha_{k}^{m} p^{m}} s_{k}^{m}$, in which $s_{k}^{m}$ depicts the desired signal and the power allocation factor of user $k$ on subcarrier $m$. $\alpha_{k}^{m}$, which satisfies $\sum_{k \in \Phi(m)} \alpha_{k}^{m}=1$, is the power allocation factor of user $k$ on subcarrier $m . z_{k}^{m}$ denotes the additive real-valued Gaussian noise with zero mean and variance $\sigma_{k}^{2}$, including the shot noise and the thermal noise. Taking the scenario in Fig. $\underline{1}$ as an example, Fig. 2 shows the principle of NOMA. To simplify the calculation, we assume that the power allocated to each subcarrier is equal, i.e., $p^{m}=P / M_{C}$, where $P$ is the total transmitted power of the MLED. Without loss of generality, users in $\Phi(m)$ are sorted based on the link gain as $h_{1} \leq h_{2} \leq \cdots \leq h_{K}$ leading to $\alpha_{1} \geq \alpha_{2} \geq \cdots \geq \alpha_{K}$. SIC is carried out at the $k$ th user to remove the signal for other users with poorer channel conditions. The message signals from users whose channel gains are stronger are treated as noise at the $k$ th user. Therefore, the achievable rate of user $k$ is denoted by

$$
R_{k}^{m}=\frac{W_{m}}{2} \log _{2}\left(1+\frac{\alpha_{k}^{m} \gamma_{k}^{m}}{\sum_{i>k} \alpha_{i}^{m} \gamma_{k}^{m}+1}\right)
$$

where $W_{m}$ is the bandwidth of subcarrier $m$, the scaling factor $1 / 2$ is due to the Hermitian symmetry, and we

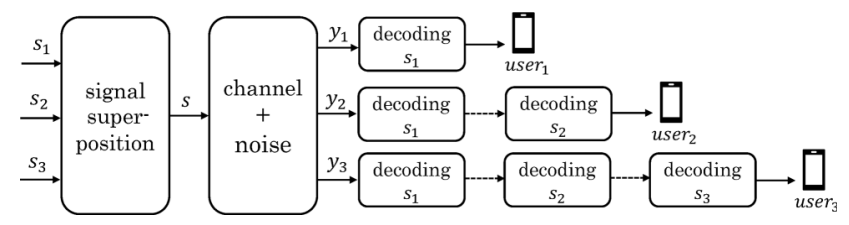

Fig. 2. Principle of NOMA. 
define $\gamma_{k}^{m}=p^{m}\left|h_{k}^{m}\right|^{2} / \sigma_{k}^{2}$. The sum rate of subcarrier $m$ can be obtained as follows:

$$
R^{m}=\sum_{k \in \Phi(m)} R_{k}^{m} .
$$

The system objective is to maximize the sum rate, which can be expressed as:

$$
\begin{gathered}
\max _{\alpha_{k}^{m}} \sum_{m \in S} \sum_{k \in \Phi(m)} \frac{W_{m}}{2} \log _{2}\left(1+\frac{\alpha_{k}^{m} \gamma_{k}^{m}}{\sum_{i<k} \alpha_{i}^{m} \gamma_{k}^{m}+1}\right), \\
\text { subject to: } \quad \sum_{i=1}^{K} a_{i}^{m} \leq 1, \\
a_{k}^{m} \geq 0 .
\end{gathered}
$$

The constraints Eqs. $(\underline{5 b})$ and $(\underline{5 c})$ ensure the limited power of each subcarrier.

To find the optimal solution of Eq. ( $\underline{5 \mathrm{a}}$ ), we need to search for all the possible combinations of users, which is not practical in real systems. Therefore, we formulate it as a matching problem to solve its suboptimal. We will first give some basic definitions and then provide our algorithm.

Matching definition: a matching $\mu$ is a mapping from the $S_{\mathrm{MEU}}$ and $S_{\mathrm{AU}}$ into the set of subcarriers such that:

1) $\mu\left(\mathrm{MEU}_{i}\right)=\mathrm{MCU}_{j}$

2) $\mu\left(\mathrm{AU}_{k}\right)=\mathrm{MCU}_{j}$ or $\mu\left(\mathrm{AU}_{k}\right)=\mathrm{ALED}_{n}$

3) $\left|\mu^{-1}\left(\mathrm{MCU}_{i}\right)\right| \leq q_{\max }$

4) $\left|\mu^{-1}\left(\mathrm{ALED}_{n}\right)\right| \leq p_{\max }$

5) $\left|\mu\left(U_{m}\right)\right| \leq 1, U_{m} \in M_{E} \cup A_{K}$

Conditions 3) and 4) state the limited number of users in $\mu^{-1}\left(\mathrm{MCU}_{i}\right)$ and $\mu^{-1}\left(\mathrm{LED}_{n}\right)$. Condition 5) represents that each MEU or AU only can match with one access point at most. The above definitions imply that $\mu$ is a many-to-one matching. Considering the operability of this matching, it assumes that all users can get served with the maximum matching number constraints.

Utility function definition: the utility function of MEU is the sum rate with MCU, which can be given by

$$
\begin{aligned}
U_{\mathrm{MEU}_{j, i}}= & \frac{W_{m}}{2} \log _{2}\left[1+\frac{\left(1-\alpha_{i}^{m}\right) \gamma_{j}^{m}}{\alpha_{i}^{m} \gamma_{j}^{m}+1}\right] \\
& +\frac{W_{m}}{2} \log _{2}\left(1+\alpha_{i}^{m} \gamma_{i}^{m}\right) .
\end{aligned}
$$

The utility function of MCU as the sum rate with users who match with him can be given by

$$
U_{\mathrm{MCU}_{i, S_{j}}}=\sum_{j \in S_{j}} R_{j}
$$

where $S_{j}$ is one of the $\mathrm{MCU}_{i}$ 's matching set.

Preference list: the preference list is a descending order list according to their preference to the other side of the users. $\mathrm{MEU}_{j}$ prefers $\mathrm{MCU}_{i}$ to $\mathrm{MCU}_{\hat{i}}$ only

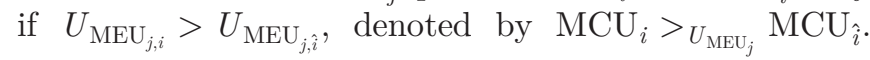

$\mathrm{MCU}_{i}$ prefers the matching set $S_{j}$ to $S_{\hat{j}}$ only if $U_{\mathrm{MCU}_{i, S_{j}}}>U_{\mathrm{MCU}_{i, S_{j}}}$, denoted by $S_{j}>_{U_{\mathrm{MCU}_{i}}} S_{\hat{j}}$.

We divide the users matching process into two phases. In the first phase, MEUs match with MCUs. During the second phase, AUs select their access points, either MCUs or ALEDs. Each phase can be seen as a many-to-one matching.

The proposed algorithms are as follows.

Phase 1: AUs' Matching Process

1. According to the received signal strength (RSS) from ALED and MLED, AUs decide to be served by which owns a stronger RSS;

2. Initialize the matched list $S_{\text {Match }}=$ $\left\{S_{\text {Match }}\left(\mathrm{ALED}_{1}\right), \ldots, S_{\text {Match }}\left(\mathrm{ALED}_{N}\right)\right\}$ for ALEDs;

3. AUs who select ALEDs send matching requests to its most preferred ALED $\hat{n}$;

4. for $n=1$ to $N$ do

5. if $\left|S_{\text {Match }}\left(\operatorname{ALED}_{\hat{n}}\right)\right| \leq p_{\max }$ then

6. $\quad$ ALED $\hat{n}$ adds it to $S_{\text {Match }}\left(\operatorname{ALED}_{\hat{n}}\right)$;

7. else

8. $\quad$ ALED $\hat{n}$ keeps the most preferred $p_{\max }$ AUs, the set of which has the max sum rate in $S_{\text {Match }}\left(\operatorname{ALED}_{\hat{n}}\right)$. The rejected one needs to require service from $\mathrm{MCU}$;

\section{9. $\quad$ end if}

\section{0. end for}

11. AUs who are rejected by ALEDs or choose MCU initially can be treated as MEUs and return to Phase 1 to complete matching process.

Phase 2: The Matching Between MCUs and MEUs

1. Initialize preference lists PL(MEU) for MEUs according to Eq. ( $\underline{6})$;

2. Initialize request lists $\mathrm{RL}(\mathrm{MCU})$ of $\mathrm{MCUs}$;

3. Initialize the matched list $S_{\text {Match }}=$ $\left\{S_{\text {Match }}\left(\mathrm{MCU}_{1}\right), \ldots, S_{\mathrm{Match}}\left(\mathrm{MCU}_{\mathrm{MC}}\right)\right\}$ for MCUs;

4. Initialize the set of unmatched MEUs $S_{\text {UnMatch }}$ to record MEUs who have not been matched;

5. while $S_{\text {UnMatch }}$ is not empty do

6. $\quad$ MEUs in $S_{\text {UnMatch }}$ send matching requests to its most preferred $\mathrm{MCU}_{\hat{i}}$ according to $\mathrm{PL}(\mathrm{MEU})$ and then remove the first $\mathrm{MCU}$ from their preference lists;

7. for $i=1$ to $M_{C}$ do

8. if $\left|S_{\text {Match }}\left(\mathrm{MCU}_{i}\right)\right|+\left|\mathrm{RL}\left(\mathrm{MCU}_{i}\right)\right| \leq q_{\max }$ then

(Algorithm continued) 
Phase 2: The Matching Between MCUs and MEUs

9. $\quad \mathrm{MCU}_{i}$ add users in $\mathrm{RL}\left(\mathrm{MCU}_{i}\right)$ into

$S_{\text {Match }}\left(\mathrm{MCU}_{\hat{i}}\right)$ and remove them from $S_{\text {UnMatch }}$;

10. else

11. $\mathrm{MCU}_{i}$ keeps the most preferred $q_{\max }$ MEUs in $S_{\text {Match }}\left(\mathrm{MCU}_{\mathrm{i}}\right) \cup \mathrm{RL}\left(\mathrm{MCU}_{i}\right)$ according to Eq. ( $\left.\underline{7}\right)$. Remove the selected ones from $S_{\text {UnMatch }}$ and add the rejected ones into $S_{\text {UnMatch }}$;

\section{2. end if}

\section{3. end for}

\section{4. end while}

Compared to the exhaustive method, the proposed algorithm applies the exhaustive method only to a very small number of users in the first phase. Assuming that there are $M$ users sending requests to ALED, the computational complexity at this stage is $\left(\mathrm{NC}_{M}^{p_{\max }}\right)$. In the second phase, each MEU makes requests $M_{C}$ times, at most. Therefore, the complexity of the grouping process for most of the remaining users in the second phase is only $\left(N M_{C}\right)$, which proves that our grouping algorithm greatly reduces computational complexity.

We compare the proposed NVUG algorithm with the traditional grouping method that the users in the $k$ th NOMA cluster are composed of the $k$ th users of each group, e.g., the first three users in the NOMA cluster, are the first user of the first group, the first user of the second group, and the first user of the third group.

Users are randomly distributed in the $12 \mathrm{~m} \times 12 \mathrm{~m}$ square area. To simplify the simulation, assuming that the number of users in each NOMA cluster is the maximum and each cluster occupies $2 \mathrm{MHz}$. The height of MLED is $5 \mathrm{~m}$, referring to the distance that a great transmission quality can be achieved for an indoor visible light

Table 1. Simulation Parameters

\begin{tabular}{lc}
\hline Parameter & Value \\
\hline Height of MLED $(\mathrm{m})$ & 5 \\
Height of ALED $(\mathrm{m})$ & 2 \\
Power of MLED $(\mathrm{W})$ & 3 \\
Power of ALED (W) & 0.3 \\
LED semi-angle $\left(^{\circ}\right)$ & 60 \\
PD FoV $\left(^{\circ}\right)$ & 60 \\
PD responsibility $(\mathrm{A} / \mathrm{W})$ & 0.4 \\
PD detection area $\left(\mathrm{cm}^{2}\right)$ & 1 \\
Reflective index & 1.5 \\
Optical filter gain & 1 \\
$N_{0}\left(\mathrm{~A}^{2} /\right.$ Hz) & $10^{-21}$ \\
\hline
\end{tabular}

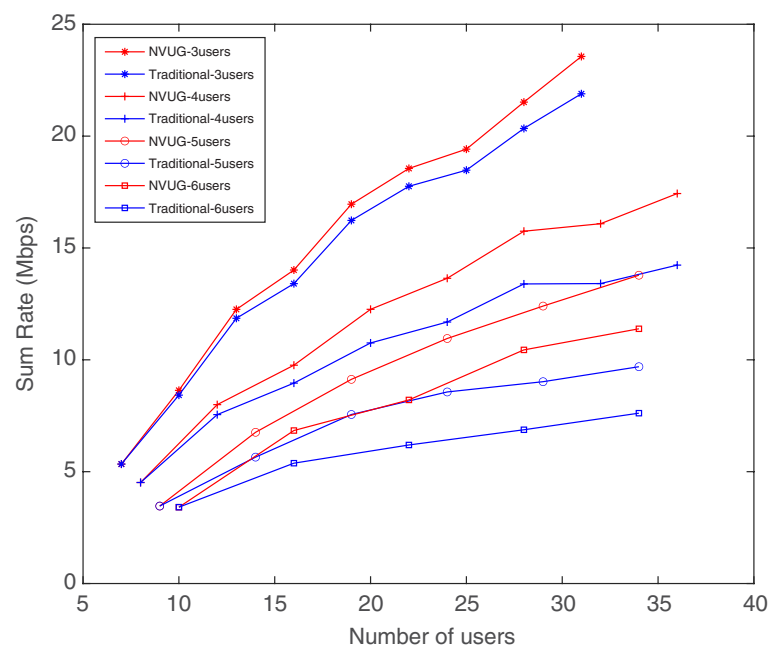

Fig. 3. Sum rate for different numbers of users.

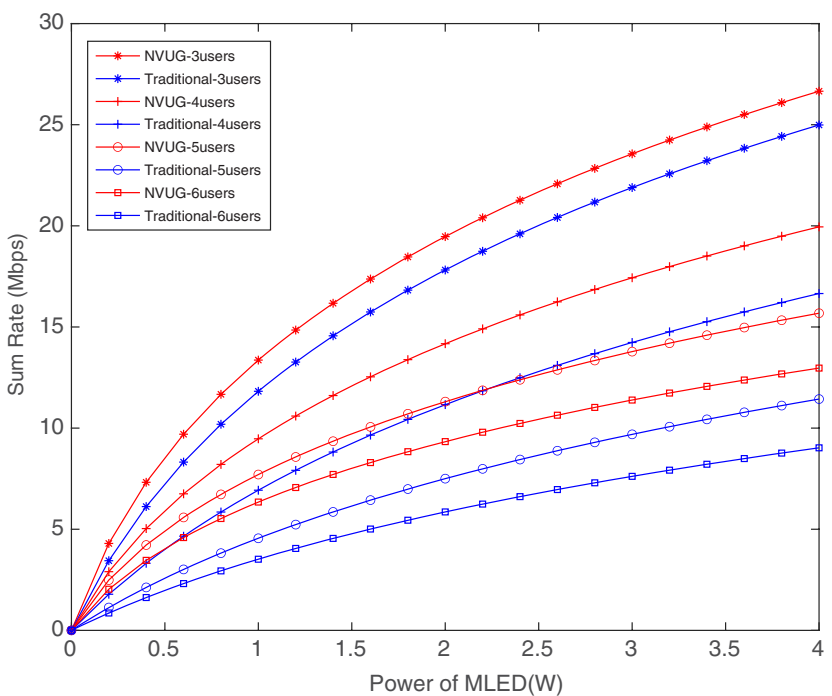

Fig. 4. Sum rate for different powers of MLED.

communication system ${ }^{[21]}$. The detailed parameters used for this simulation are summarized in Table $\underline{1}$. Moreover, a fixed power allocation scheme was chosen to allocate the power for each user, and the transmission power for the $k$ th sorted user is set to $P_{k}=\alpha P_{k-1}, \alpha=0.3$, which has the best bit error rate average performance $\frac{[22]}{}$.

Figure 3 shows that the proposed user grouping scheme can get a higher achievable sum rate than the traditional grouping scheme does, which implies that the NVUG does provide a better user-grouping method. Moreover, as the number of users in each NOMA cluster increases, the sum rate difference between the two grouping methods is more obvious, which benefits from the cost of computational complexity of the proposed grouping scheme. It also can be seen from the simulation results in Fig. 4 that increasing the transmission power of the MLED also improves the sum rate of this system because higher power essentially means a higher signal strength. But the strength of the 
interference signal will also improve, so the increase of the achievable sum rate is gradually flattened.

In this Letter, a user-grouping scheme that is based on matching theory is proposed to solve the multi-user grouping problem for indoor NOMA-VLC heterogeneous networks. Comparing it with the traditional grouping scheme proves that the proposed scheme can obtain a higher system achievable sum rate through simulation. Simulation results also show that, as the number of users in each NOMA cluster increases, the proposed grouping scheme can achieve better performance. Future research can further consider the impact of user mobility, more complex heterogeneous environments such as integration with traditional RF networks, and environment interference on user grouping.

This work was supported in part by the National Key R\&D Program of China (No. 2017YFB0403605), the National Natural Science Foundation of China (No. 61801165), and the National 973 Program of China (No. 2013CB329205).

\section{References}

1. Y. Ji, J. Zhang, X. Wang, and H. Yu, Sci. China Inf. Sci. 61, 12 (2018).

2. Y. Ji, J. Zhang, Y. Xiao, and Z. Liu, China Commun. 16, 5 (2019).

3. H. Elgala, R. Mesleh, and H. Haas, IEEE Commun. Mag. 49, 56 (2011).

4. A. Jovicic, J. Li, and T. Richardson, IEEE Commun. Mag. 51, 26 (2013).

5. N. Chi and M. Shi, Chin. Opt. Lett. 16, 120603 (2018).

6. X. Huang, F. Yang, and J. Song, Chin. Opt. Lett. 17, 100002 (2019).

7. E. W. Lam and T. D. C. Little, Chin. Opt. Lett. 17, 030604 (2019).
8. D. Karunatilaka, F. Zafar, V. Kalavally, and R. Parthiban, IEEE Commun. Surv. Tutorials 17, 1649 (2015).

9. H. Haas, L. Yin, Y. Wang, and C. Chen, J. Lightwave Technol. 34, 1533 (2016).

10. H. Marshoud, S. Muhaidat, P. C. Sofotasios, S. Hussain, M. A. Imran, and B. S. Sharif, IEEE Wireless Commun. 25, 82 (2018).

11. L. Dai, B. Wang, Y. Yuan, S. Han, C.-L. I, and Z. Wang, IEEE Commun. Mag. 53, 74 (2015).

12. W. Shin, M. Vaezi, B. Lee, D. J. Love, J. Lee, and H. V. Poor, IEEE Commun. Mag. 55, 176 (2017).

13. Z. Ding, Z. Yang, P. Fan, and H. V. Poor, IEEE Signal Process. Lett. 21, 1501 (2014).

14. R. C. Kizilirmak, C. R. Rowell, and M. Uysal, in 4th International Workshop on Optical Wireless Communications (IWOW) (2015), p. 98.

15. Y. Gu, W. Saad, M. Bennis, M. Debbah, and Z. Han, IEEE Commun. Mag. 53, 52 (2015).

16. X. Zhang, Q. Gao, C. Gong, and Z. Xu, IEEE Commun. Lett. 21, 777 (2017).

17. Y. Fu, Y. Hong, L. Chen, and C. W. Sung, IEEE Photonics Technol. Lett. 30, 1218 (2018).

18. Y. Han, X. Zhou, L. Yang, and S. Li, in International Conference on Computing, Networking and Communications (ICNC) (2018), p. 480 .

19. H. Zhang, N. Liu, K. Long, J. Cheng, V. C. M. Leung, and L. Hanzo, IEEE J. Sel. Areas Commun. 36, 658 (2018).

20. L. Zeng, D. C. O’Brien, H. L. Minh, G. E. Faulkner, K. Lee, D. Jung, Y. Oh, and E. T. Won, IEEE J. Sel. Areas Commun. 27, 1654 (2009).

21. B. Fahs, J. Chellis, M. J. Senneca, A. Chowdhury, S. Ray, A. Mirvakili, B. Mazzara, Y. Zhang, J. Ghasemi, Y. Miao, P. Zarkesh-Ha, V. J. Kooson, and M. M. Hella, IEEE Photonics Technol. Lett. 28, 2846 (2016).

22. H. Marshoud, P. C. Sofotasios, S. Muhaidat, G. K. Karagiannidis, and B. S. Sharif, IEEE Trans. Wireless Commun. 16, 6350 (2017). 